\title{
NEW INSERTION DEVICES FOR ELETTRA
}

\author{
B. Diviacco, R. Bracco, D. Millo, M. Zalateu, D. Zangrando, R.P. Walker \\ Sincrotrone Trieste, s.s.14, km 163,5, 34012 Trieste, ITALY
}

\section{INTRODUCTION}

After completion of a series of variable polarization undulators, presently under commissioning [1,2], three new insertion devices are being developed for ELETTRA. A short $(1 \mathrm{~m})$ undulator has been built, and will soon be installed in a dispersive straight section of the ring. A $3.5 \mathrm{~T}, 60 \mathrm{~mm}$ period superconducting multipole wiggler is also under construction. The third device is a full length $(4.5 \mathrm{~m})$ Figure- 8 undulator, which is designed to produce low energy linearly polarized photons with reduced heat load on the beamline optical elements.

\section{SHORT ID PROTOTYPE}

In order to increase the number of insertion devices installed in the storage ring and since all eleven ID straight sections have already been defined and will be completed by 2002 , the possibility of installing short insertion devices, with a maximum length of $1 \mathrm{~m}$ in dispersive regions of the machine lattice has been studied [3]. The predicted brilliance for a range of different short devices is compared in fig. 1 with that of a bending magnet source.

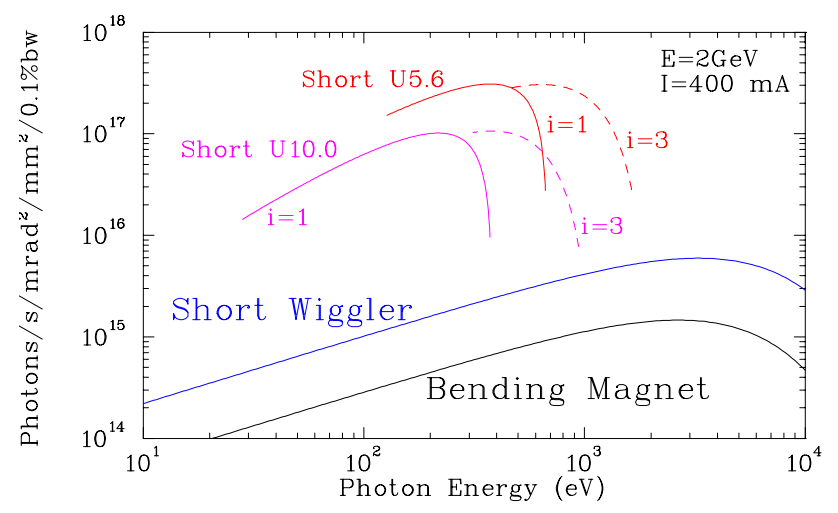

Figure 1: Brilliance of two short undulators (the U5.6 prototype and a generic $10 \mathrm{~cm}$ period device), a short wiggler $\left(15 \mathrm{~cm}\right.$ period, $\left.\mathrm{B}_{0}=1.6 \mathrm{~T}\right)$ and a bending magnet.

To test this new idea, the construction and the installation of a short-ID prototype with a period of 56 $\mathrm{mm}$ was approved at the beginning of 2000 . The elliptical vacuum chamber, constructed from stainless steel $316 \mathrm{LN}$, with an internal (external) diameter of 18 (22) $\mathrm{mm}$ (consistent with a minimum undulator gap of $23 \mathrm{~mm}$ ) was installed in March 2001.
The prototype short insertion device, which makes use of existing magnetic arrays, has recently been completed (see fig.2) and will be installed in June this year. The carriage has been constructed by ICAP, Italy. Each stainless steel beam, supporting the magnetic arrays is moved by only one screw-jack and DC motor (instead of 2, as used for the other carriages installed in ELETTRA). This solution has the advantage of reducing the complexity of the drive and control system, avoiding gap tapering in case of control system faults. Furthermore, the cost is significantly reduced compared to the previously used 4-axes structure.

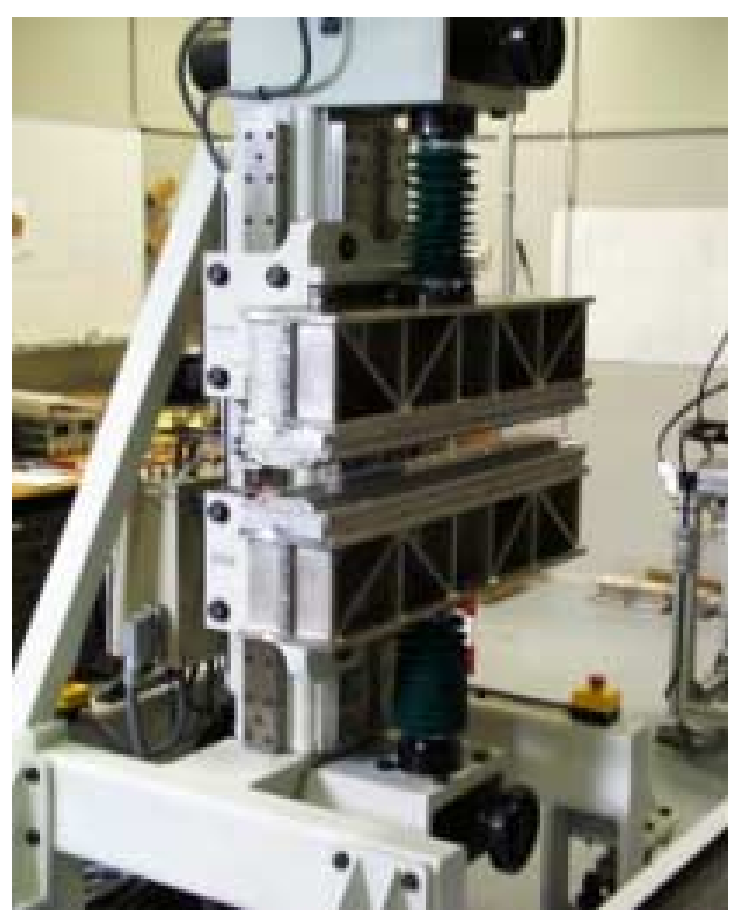

Figure 2: Photograph of the new short ID, in the magnetic measurement laboratory.

The drive system has been designed in order to set the magnetic gap with an accuracy of $20 \mu \mathrm{m}$ under a maximum magnetic load of $40 \mathrm{kN}$. The parallelism of the beams is guaranteed by the side and front roller bearings which travel on the guide rails. Measurements using a specially constructed tool, have shown that this system is capable of containing the gap tapering to less than 60 (20) $\mu \mathrm{Rad}$ with a load of 3.75 (1.25) $\mathrm{kN}$ applied to one beam 
extremity. In the usual situation however the magnetic load is always centred and so the possible tapering is much smaller. In any case, for safety reasons, two linear encoders (resolution $1 \mu \mathrm{m}$ ) have been installed, one on each side of the structure. Using these two encoders, the parallelism of the beams is constantly monitored and the motion is stopped in case of any excessive disalignment. We are presently evaluating the possibility of using this type of carriage for undulators with beam length up to $2 \mathrm{~m}$.

\section{SUPERCONDUCTING WIGGLER}

For the second Diffraction beamline a higher flux and brightness source was requested compared to the 57-pole 1.6 T multipole wiggler (W14.0) currently in use on the existing Diffraction beamline. Following the basic idea of a superconducting multipole wiggler device being developed at MAX-lab [4] a preliminary design study [5] concluded that such a device was also an ideal source for photons in the $10-25 \mathrm{keV}$ range in ELETTRA. The main parameters of the device are given in Table 1.

Table 1. Main superconducting wiggler parameters

\begin{tabular}{|l|l|}
\hline Period length & $60 \mathrm{~mm}$ \\
\hline Peak field & $3.5 \mathrm{~T}$ \\
\hline Total no. of poles & 49 \\
\hline Pole sequence & $1 / 4,-3 / 4,1,-1 \ldots 1,-3 / 4,1 / 4$ \\
\hline Internal aperture & $81 \mathrm{~mm}(\mathrm{H}) \times 10.7 \mathrm{~mm}(\mathrm{~V})$ \\
\hline Total power & $17.2 \mathrm{~kW}(2 \mathrm{GeV}, 400 \mathrm{~mA})$ \\
\hline
\end{tabular}

Figure 3 shows that the improved flux compared to the existing wiggler is a factor of $3(14)$ at $12.5(25 \mathrm{keV})$. A further significant improvement is gained from the smaller source size resulting from the shorter wiggler length (1.4 $\mathrm{m}$ instead of $4.5 \mathrm{~m})$ [5].

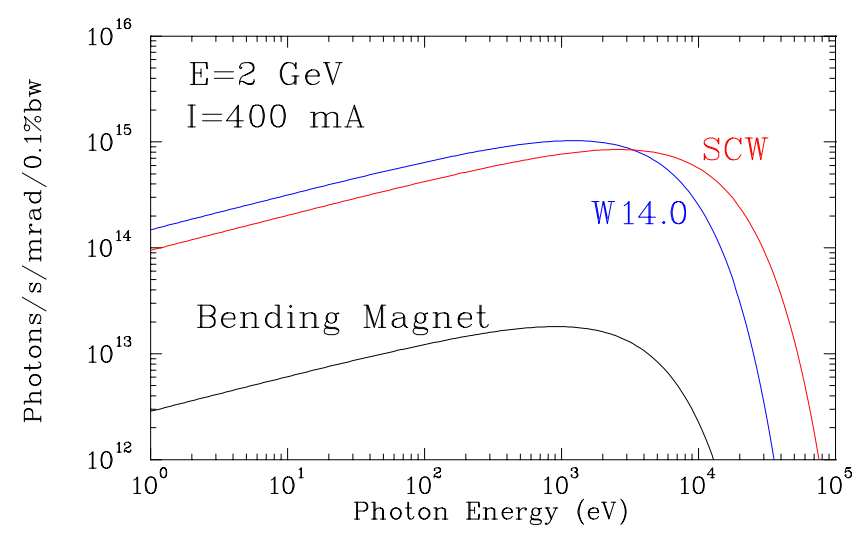

Fig. 3. Photon flux from the superconducting wiggler, W14.0 and bending magnet sources.
Following a tender exercise, a contract was subsequently placed with the Budker Institute of Nuclear Physics for its detailed design and manufacture, which is now in progress. The wiggler has a cold-bore design with internal $20 \mathrm{~K}$ copper liner to efficiently absorb the thermal load from scattered photons and r.f. heating.

\section{FIGURE-8 UNDULATOR}

A new undulator is presently under construction as the radiation source for the Inelastic Ultra-Violet Scattering (IUVS) beamline, which requires a high flux of linearly polarized photons in the 5 to $10 \mathrm{eV}$ range. To overcome the problems due to the very high on-axis power density that a conventional undulator would generate, a Figure- 8 structure $[6,7]$ has been adopted. The undulator is made of six permanent magnet arrays (see fig. 4) that generate a vertical field component of period $\lambda_{0}$ (center blocks) and a horizontal field component of period $2 \lambda_{0}$ (side blocks).

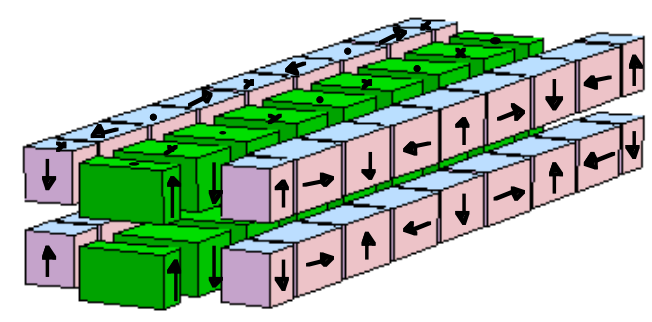

Figure 4: geometry of the Figure-8 undulator

With an appropriate phasing between the central and side arrays, the resulting electron motion looks like a figure-of-eight when projected in the transverse plane, hence the name. More interestingly, the angular distribution of the emitted power is peaked off-axis so that a suitable pinhole can filter-off most of the unwanted power, thus reducing by a large factor the heat load on the beamline optical elements.

The chosen undulator parameters are listed in Table 2 below. Constraints on the total emitted power, the maximum vertical deflection angle and the harmonic content of the spectrum (see discussion below) were carefully considered during parameter optimization.

Table 2: Main undulator parameters

\begin{tabular}{|l|l|}
\hline magnetic material & $\mathrm{NdFeB}(\mathrm{Br}=1.28 \mathrm{~T})$ \\
\hline period length $(\mathrm{mm})$ & 140 \\
\hline number of periods & 32 \\
\hline minimum gap $(\mathrm{mm})$ & 19 \\
\hline Bxo, Byo $(\mathrm{T})$ at minimum gap & $0.13,0.72$ \\
\hline Deflection parameters Kx, Ky & $3.4,9.4$ \\
\hline Total power $(\mathrm{KW})$ & $2.5(2 \mathrm{GeV}, 400 \mathrm{~mA})$ \\
\hline
\end{tabular}


The computed power density angular distribution (at minimum gap) can be seen in fig. 5, which shows the very low central values achieved by this design.

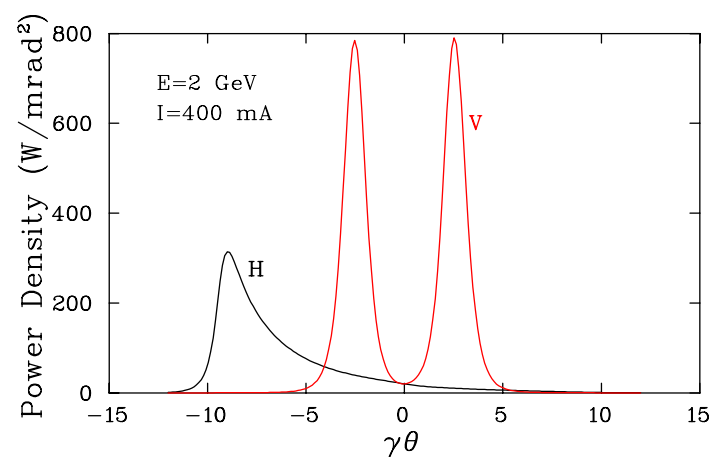

Figure 5: Angular distribution of the power density along the horizontal $(\mathrm{H})$ and vertical $(\mathrm{V})$ directions.

The strong suppression of the power transmitted to the beamline is demonstrated in fig. 6 where a comparison is made with a conventional vertical-field undulator having the same fundamental photon energy, period length and number of periods.

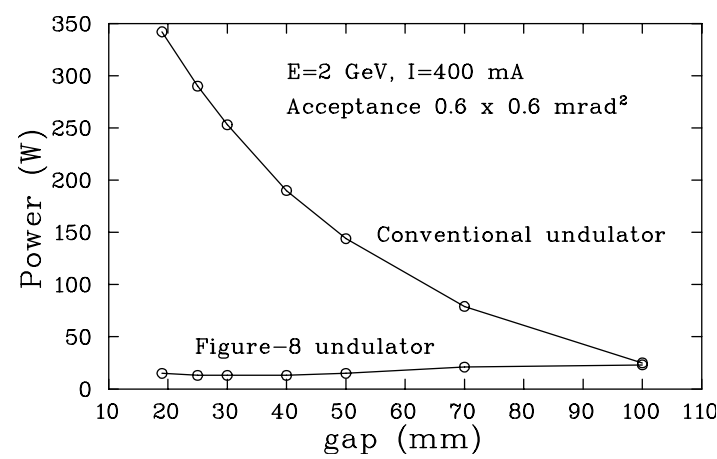

Figure 6: Power within the nominal beamline acceptance angle for the new undulator and a conventional undulator.

Due to the large emission angle of the fundamental compared to that of the higher-order harmonics (which in such a high $\mathrm{K}$ device carry most of the energy) efficient power filtering is achieved with no significant penalty on the useful photon flux. This is shown in fig. 7 where the spectral intensity produced by the figure- 8 undulator is compared to that of standard undulator with the same period length and fundamental photon energy. Note the presence of semi-integer harmonics, characteristic of this type of magnetic structure [7]. For the practical applications foreseen for this device, only the harmonic $1 / 2$ is potentially harmful, because it cannot be efficiently filtered by the optical system. However, due to the relatively weak horizontal field strength, its intensity relative to that of the fundamental is sufficiently low that it will produce negligible effects on the spectral purity of the monochromatized beam.
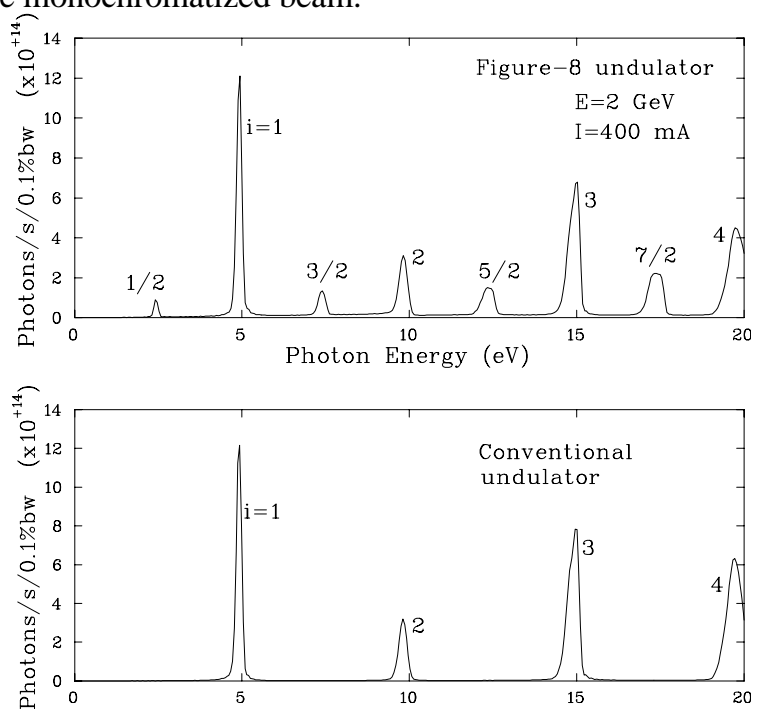

Figure 7: Pinhole spectrum of the new (upper graph) and a conventional undulator (lower graph).

For practical reasons, the undulator will be segmented in two 16 periods units. Phasing between the two modules is achieved by simply allowing a small $(\sim 1 \mathrm{~mm})$ separation between the end blocks of the central arrays. In this way the vertical field periodicity is almost maintained along the complete device, producing negligible effects on the spectral distribution at the lower order harmonics. More details on this and other techological issues (such as those related to the non-unit permeability of the magnetic material, the choice of the termination type, the foreseen methods for correcting random and sistematic magnetic field errors) can be found in a separate document [8].

\section{REFERENCES}

[1] B. Diviacco et al., proc. EPAC 2000, pag. 2322.

[2] L. Tosi et al., proc. EPAC 2000, pag. 2349.

[3] L. Tosi, R.P.Walker, Sincrotrone Trieste Internal Report ST/SL-01/01 (April 2001).

[4] G. LeBlanc, E. Wallén and M. Eriksson, proc. EPAC 2000, p. 2334.

[5] R.P. Walker, Sincrotrone Trieste Internal Note in preparation.

[6] T. Tanaka, H. Kitamura, Nucl. Instr. and Meth. in Phys. Res. A 364 (1995) p. 368.

[7] T. Tanaka, H. Kitamura, J. Synchrotron Rad. (1996) 3, p. 47.

[8] B.Diviacco, Sincrotrone Trieste Internal Note in preparation. 